\title{
Comparison of microvascular decompression and two isocenters gamma knife for the treatment of trigeminal neuralgia caused by vertebrobasilar compression
}

\section{Zhen Zhao}

Wuhan Union Hospital

Songshan Chai

Wuhan Union Hospital

Jiajing Wang

Wuhan Union Hospital

Xiaobing Jiang

Wuhan Union Hospital

Chuansheng Nie

Wuhan Union Hospital

Hongyang Zhao ( $\square$ hyzunion@163.com )

Wuhan Union Hospital https://orcid.org/0000-0003-3895-614X

\section{Research Article}

Keywords: Trigeminal neuralgia, Vertebrobasilar dolichoectasia, Microvascular decompression, Gamma Knife, Pain relief

Posted Date: April 16th, 2021

DOl: https://doi.org/10.21203/rs.3.rs-407050/v1

License: (c) (i) This work is licensed under a Creative Commons Attribution 4.0 International License. Read Full License 


\section{Abstract}

Objective: Vertebrobasilar dolichoectasia (VBD) is one of the uncommon causes of trigeminal neuralgia (TN). The main surgical treatments for TN caused by VBD (VBD-TN) are invasive microvascular decompression (MVD) and mini-invasive Gamma Knife radiosurgery (GKRS). However, the therapeutic effects of the two methods have not been clinically reported, so this study evaluated the outcomes of MVD and GKRS for patients with VBD-TN.

Methods: The retrospective study of patients diagnosed with VBD-TN in Wuhan Union Hospital was performed from March 2011 to March 2019. A total of 80 patients were included in this study, and they were divided into the MVD group $(n=46)$ and GKRS group $(n=34)$ according to the surgical methods. Among patients in the GKRS group, all performed by two isocenters gamma knife. The imaging data, intraoperative findings, outcomes, and complications of the two groups were analyzed and compared.

Results: Patients who underwent MVD were younger than patients who underwent GKRS (median ages were 61.1 and 65.4 years old, $p=0.03$ ). The average follow-up was 61.1 months for the MVD group and was 56.8 months for the GKRS group. The favorable outcomes (BNI score I-II) occurred in $97.8 \%$ of patients treated with MVD and in $78.9 \%$ of patients treated with GKRS $(P=0.009)$. The favorable outcomes in the percentage of patients after MVD 1,3,5 and 7 years were $95.7 \%, 85.1 \%, 74.2 \%$, and $74.2 \%$. The corresponding percentages after GKRS were $76.5 \%, 66.2 \%, 56.6 \%$ and $47.2 \%$, respectively $(P=0.031)$. The postoperative complications (except facial numbness) in the MVD group were higher than those in the GKRS group $(P=0.036)$, but the incidence of new and worsening facial numbness was higher in the GKRS group $(P<0.001)$.

Conclusions: For VBD-TN, MVD is superior to GKRS in obtaining and maintaining favorable outcomes, but it also comes with more complications other than facial sensation. Treatment choice can be tailored to a patient's unique condition and wishes.

\section{Introduction}

Trigeminal neuralgia (TN) is mainly characterized by recurrent paroxysmal electric shock-like and acupuncture-like pain in the distribution area of the trigeminal nerve that composed of the eye branch (V1), the maxillary branch (V2), and the mandibular branch (V3), which usually lasts for a few seconds[11, 37, 47]. The actions, including brushing teeth, gargle, washing face, chewing, and other activities, especially when eating cold or hot food, can induce TN. The cause of primary TN that trigeminal nerve in the cerebellopontine angle area is compressed by the responsible blood vessels has been reported, resulting in demyelination of trigeminal nerve and short circuits between the afferent and efferent nerves impulse[27]. Common offensive blood vessels include superior cerebellar artery (SCA), anterior inferior cerebellar artery (AICA), mixed arteriovenous, posterior inferior cerebellar artery (PICA). However, TN caused by the vertebrobasilar artery accounts for approximately $2 \%-7 \%$ of all types of TN, which is relatively rare[4, 8, 24,34]. 
Drug treatment should also be performed first once VBD-TN was diagnosed like TN caused by other blood vessels. The commonly used drugs include carbamazepine, oxcarbazepine, and phenytoin, and so on. When drugs cannot control the pain symptoms, surgical treatment should be considered[2]. There are various surgical techniques that have been used for these cases, including MVD, GKRS, percutaneous glycerol lesions, percutaneous balloon compression, radiofrequency thermocoagulation. MVD may be considered as the most definite and durable treatment method for VBD-TN[7]. However, the postoperative outcomes of patients with VBD-TN, reported by some studies, are relatively unsatisfactory in terms of recurrence and complications[45]. These unsatisfactory outcomes may be attributed to large volume, stiff vessel walls, and high arterial flow, making it less mobile than small arteries (such as superior cerebellar artery reported as the most common offending artery) $[5,23,25]$. Considering these, coupled with the extremely high risk of craniotomy, a lot of surgeons may prefer other surgical treatments, for instance, the mini-invasive GKRS is the most used alternative technology, especially for elderly frail patients with organ dysfunction[37]. However, single isocenter GKRS treatment of TN has poor pain relief, especially for trigeminal neuralgia caused by compression of big blood vessels[13]. It has been reported that two isocenter GKRS can completely cover the offensive blood vessels and can significantly improve the pain relief rate[1].

To the knowledge of previous studies, both MVD and two isocenter GKRS can effectively relieve the pain of patients for VBD-TN, but the effectiveness and safety of the two techniques that worthy of research have not been reported. Therefore, we have retrospectively compared the treatment outcomes of two techniques for patients with VBD-TN.

\section{Materials And Methods}

\section{Study population}

This study was conducted in accordance with the Declaration of Helsinki and approved by our institutional review board, however, the informed consent of patients was not required on account that was a retrospective study. Eighty consecutive patients with VBD-TN diagnosed between March 2011 to March 2019 were included in the study. All of them performed MVD or GKRS in the Union Hospital of Huazhong University of Science and Technology, Wuhan, China. In the same period, 960 patients diagnosed as primary TN with other offensive blood vessels including AICA, PICA, SCA, and petrosal vein were excluded. Moreover, patients diagnosed with secondary TN caused by tumor and inflammation in the cerebellopontine angle area were excluded, too. All patients were informed for the treatment plans of VBD-TN and the expected effects and complications of various treatment plans through interviews, and the final choices of the surgical method were decided by the patients. The included patients were divided into MVD group ( $n=46)$ and two isocenter GKRS group $(n=34)$ according to the surgical method. The average age was $61.1 \pm 11.25$ years in the MVD group and 65.4 \pm 9.34 in the GKRS group, ranging from 30 to 70 years old and 38 to 75 years old, respectively. 
All patients were examined by using the 3.0 T magnetic resonance imaging (MRI) scanner (Magnetom Trio; Siemens AG, Erlangen, Germany) before surgery. The diagnostic criteria of VBD-TN: the maximum diameter of the vertebrobasilar artery exceeds $4.5 \mathrm{~mm}$, the basilar artery bifurcates above the level of the suprasellar cistern or near the side edge of the slope or the dorsal saddle, and the tortuous expansion of the vertebrobasilar artery and the symptomatic side trigeminal nerve Pressure.

Patients' clinical records were reviewed, including clinical characteristics, pain laterality, pain distribution, neuroradiological presentations and their duration, intraoperative findings, treatment outcomes, and postoperative complications.

\section{MVD}

The patients were taken to the contralateral position after general anesthesia, the dura mater was cut along the lower edge of the transverse sinus and the posterior edge of the sigmoid sinus and then was suspended to release the appropriate amount of cerebrospinal fluid to provide space for operation. Through arachnoid dissection and slight elevation of the cerebellum, the neurovascular conflicts located at trigeminal nerve root entry zone (REZ) were carefully observed using an operating microscope, and a 45-degree endoscope was used when necessary. It was found that all trigeminal nerve roots, with varying degrees, were compressed by the vertebrobasilar artery.

In order to decompress the vertebrobasilar artery, however, we use two different MVD techniques: interposition method and the transposition method. In the Interposition subgroup, we introduced the chopped Teflon felt implant into the conflicting neurovascular area between the artery and nervous structures, thereby separating the VBD from the trigeminal nerve. While in the transposition subgroup, the proximal part of the vertebrobasilar artery was moved ventrally and cranially through the gap between the IX and VII-VIII nerves, and then fixed on the nearby petrous bone wall with biomedical glue. It is worth noting that the perforating arteries should be protected to avoid secondary damage when suspended and to avoid twisting into angles for the responsible blood vessels[22, 25]. Furthermore, the biomedical glue was strictly forbidden to disperse into subarachnoid space. (Figure 1)

\section{Radiosurgical procedure}

All patients of GKRS group were treated by using Leksell Model B Gamma Knife (Elekta Instruments, Stockholm, Sweden). The Leksell Model G stereotactic frame was installed on the skull after the patient received local anesthesia. Then 3.0T MRI was taken to clarify the relationship between the trigeminal nerve and its surrounding blood vessels, 3D-TOF-MRA and 3D-FIESTA sequence, $1 \mathrm{~mm}$ slice thickness, no interval positioning scan were obtained. A $4 \mathrm{~mm}$ isocenter was placed adjacent to the trigeminal nerve root entry zone and another isocenter at the distal cisternal segment. Due to the close proximity of the two targets, the $50 \%$ dose curves of the two isocenters often partially overlap and are connected to form a "cucurbit" shape. If the cisternal segment of trigeminal neuralgia is severely moved by the vertebrobasilar artery and is difficult to be clearly displayed, one target is placed in the REZ area as much as possible according to the recognition situation, and the other target is often placed 2-3 $\mathrm{mm}$ behind the trigeminal 
nerve enters Meckel's cavity. The maximum central dose was $80-90 \mathrm{~Gy}$, the isodose curve is $50 \%$, and the dose of the pontine surface does not exceed 20 Gy (Figure 2).

\section{Follow-up and evaluation of treatment outcome}

Clinical follow-up information was obtained by regular telephone interviews or by questionnaires. Barrow Neurological Institute pain intensity score (BNI) was used to assess patient pain relief. BNI score I, no pain, no medication; II, occasional pain, no medication; III, some pain, adequately controlled with medications; IV, some pain, not adequately controlled with medications; $\mathrm{V}$, severe pain or no pain relief. Patients' pain intensity was assessed preoperatively, and at 1, 3, 6, and 12 months postoperatively and then yearly thereafter. We considered BNI scores I to II as representing favorable outcomes (pain-free or pain occasionally), and yet BNI scores III to V was defined as unfavorable outcomes. The BNI score of I was regarded as the pain-free outcome. Furthermore, we also sought patients if there any complications such as facial numbness, dry eyes, hearing loss, diplopia, and so on.

\section{Statistical analysis}

All statistical analyses were accomplished using SPSS software of version 25.0 (IBM Corp., Armonk, New York, USA). P-values of less than 0.05 were considered to indicate statistical significance. The continuous variables are reported as mean \pm standard deviation (SD) or range, whereas categorical variables are presented as median and quartiles or by absolute and relative frequencies. Statistical testing utilized nonparametric tests with Mann-Whitney $U$ test and Kruskal-Wallis test for continuous variables, and the chisquare test or Fisher exact test for categorical variables. The correlation of treatment outcomes with clinical factors (age, sex, pain distribution, duration of symptoms, hypertension, median time to pain relief) was calculated using Pearson's Chi-square test. The interval of maintaining favorable outcomes that was defined as the time between no pain and detection of some pain controlled by drug (BNI III) or until the last follow-up was assessed using the Kaplan-Meier method and compared by the log-rank test for the MVD group and GKRS group.

\section{Results}

\section{The characteristic of patient population}

The demographic and clinical characteristics of the patient population are shown in Table 1. There were 46 patients in the MVD group and 34 patients in the GKRS group included in this study, respectively. The average age of all participants was 62.93 years old, and the proportion of male patients was $65 \%$. The pain side is most common on the left side, accounting for approximately $62.5 \%$ of cases. The branches of pain distribution are the most common in $\mathrm{V} 2+\mathrm{V} 3$, accounting for about $37.5 \%$. Demographic data showed that the age of patients performed by MVD was younger than the patients performed by GKRS $(P=0.03)$. But there was no significant difference in gender, pain side, basal BNI score, history of prior operation, hypertension, pain distribution, duration of symptoms between the two groups of patients (all $\mathrm{P}>0.05)$. 


\section{Pain relief outcomes}

In terms of initial favorable outcomes (BNI score I to II), All but one patients reached immediately initial favorable outcome after surgery in MVD group. Among the forty-five patients, forty-three patients had BNI score I, two patients had BNI score II. The remaining one patient had BNI score III. While there were only twenty-seven patients who reached initial favorable outcomes in the GKRS group, twenty-four patients of them had BNI I, three patients of them had BNI II. The remaining patients had unfavorable outcomes, three of them had BNI score III, one of them had BNI score IV, three of them had BNI score V. It is obvious that the MVD group (97.8\%) is statistically superior to the GKRS group (78.9\%) about the rate of initial favorable outcomes ( $P=0.005$, Table 2$)$. Moreover, the time required to obtain initial favorable outcomes in the GKS group was significantly longer than that in the MVD group ( 8 weeks vs 0 day, $P=0.009$, Table 2)

At the last follow-up, there were 38 patients (82.6\%) in MVD group and 20 patients (58.8\%) in GKRS group maintained favorable outcomes. The MVD group showed significantly better compared with the GKRS group in terms of the ability to maintain favorable outcomes ( $p=0.03$, Table 2$)$. In order to further observe the long-term effect of the two surgical methods, Kaplan-Meier survival analysis was performed, which showed that the MVD group showed significantly superior compared with the GKRS group for the time of maintaining favorable outcomes (Figure $3, p=0.031$, log-rank test). The favorable outcomes in the percentage of patients after MVD 1,3,5 and 7 years were $95.7 \%, 85.1 \%, 74.2 \%$, and $74.2 \%$. The corresponding percentages after GKRS were $76.5 \%, 66.2 \%, 56.6 \%$ and $47.2 \%$, respectively.

In the MVD group, a comparison of the influencing factors between patients with favorable outcomes (BNI I-II) and patients with unfavorable outcomes (BNI III-IV) found that there were no statistically significant differences in age, gender, pain laterality, surgical history, pain distribution, preoperative BNI scores, offensive blood vessels and hypertension (all $P>0.05$ ), only the MVD technique was significantly different $(P=0.037)$. In the GKS group, only new or worsening facial numbness after surgery was significantly different between the group with favorable outcomes and the group with unfavorable outcomes.

\section{Post-operation numbness}

The Table 2. demonstrated that patients who received MVD significantly higher than patients who received GKRS in terms of new or worse numbness (three patients in the MVD group vs sixteen patients in the GKRS group, $P<0.001)$. Among the three patients in the MVD group, two patients thought the numbness was not bothersome, and another suffered a little bothersome numbness, but no patients complained the numbness was very bothersome. In the GKRS group, however, most of them suffered numbness without bothersome (eleven patients, 32.4\%) and with a little bothersome (two patients, 5.9\%), only two patients complained the numbness was very bothersome.

\section{Other complications}


The comparison of other complications between MVD and GKRS group was shown in Table 2. Otherwise, which revealed the MVD group was obviously higher than the GKRS group (eleven patients in the MVD group vs two patients in the GKRS group, $P=0.036$ ). These included three patients who experienced hearing loss with the median time of 2 years, taste hypoesthesia and wound infection were complained by two patients each other, and newly development of dry eyes, facial palsy, diplopia and cerebrospinal fluid leakage were all one patient in the MVD group. Among them, patient with dry eye did not require special intervention; patient with diplopia was completely resolved spontaneously within 2 months without special treatment; patients with facial nerve palsy were treated with mecobalamin tablets (500 mg, 3 times a day for 1 month), and the palsy subsided completely; the patients with cerebrospinal fluid leakage healed after surgical repairing; of the 2 cases of hypogeaia, one patient resolved spontaneously, and the other persisted for a long time. All 3 patients of hearing loss occurred on the side of TN. They received a comprehensive drug treatment including nimodipine, methylprednisolone and neurotrophic agents. Two of them also received adjuvant hyperbaric oxygen therapy. Their symptoms improved to varying degrees: one patient returned to normal hearing, while the other two cases developed permanent mild hearing impairment. But yet, in the GKRS group, only 2 people complained about newly development of dry eyes, and none of them required special intervention.

\section{Discussion}

VBD, a rare arterial disease caused by vertebrobasilar tortuosity and expansion, can lead to obvious compression for the trigeminal nerve, which brings about the occurrence of TN. Some studies have reported that VBD-TN is more common in elderly male patients, usually on the left side, and tends to suffer from arterial hypertension[15, 25, 29, 40,43]. In our study, the clinical baseline characteristics in the MVD and GKRS groups were consistent with those findings. These characteristics can be explained by the general association of these three factors with atherosclerosis[33]. It seems that long-term high blood flow has an impact on hemodynamics through atherosclerosis, which can cause arteries to bend and tortuous[34]. The vasodilatation may be related to the structural defects of the arterial wall in the inner elastic layer[36]. As for the left side was the dominant pain side, which can be attributed to the asymmetry of the origin of the vertebrobasilar artery and hemodynamic factors [17, 25]. Our other findings that the common pain distribution were single branch and $\mathrm{V} 2+\mathrm{V} 3$ branches agree with those reported by Maarbjerg et al. [30] . Those could be related to the position distribution of sensory fibers in the trigeminal nerve root.

The studies are still based on case reports and case sequences of a single regimen due to its relative rarity, and there is a lack of research on larger samples and comparison of treatment methods. Therefore, there is no standard technology to deal with the VBD-TN. Drug treatment is the preferred treatment, but when the pain is out of control, other forms of treatment are needed, such as $\operatorname{MVD}[4,9]$, peripheral nerve resection[33], peripheral nerve alcohol injection[28], percutaneous glycerol lesions[16, 44], percutaneous balloon compression[3, 6], GKRS[11, 49] and radiofrequency thermocoagulation[21, 35]. MVD and GKRS are the more common treatment methods used in clinical practice nowadays due to acquiring good painrelief outcomes. MVD surgery variety of forms, in general, there are two different modes for separating 
the offending VBA, including the interposition method and transposition method[9]. Therefore, MVD can fully push the vertebrobasilar artery away from the nerve by inserting Teflon felt implant into the conflicting neurovascular area or fixing the blood vessels during the operation, thereby eliminating the neurovascular conflicts. GKRS is a minimally invasive radiotherapy for the treatment of VBD-TN. According to the number of targets in the radiology, it can be divided into single isocenter[42], two isocenters[39] and multiple isocenters[11]. The benefits of GKRS for primary TN have been recognized, furthermore, many patients with recurrent pain can relieve pain effectively after receiving the second GKRS treatment[10,14,37]. This phenomenon led us to speculate that for some TN patients, especially obvious in patients with VBD-TN due to their wider neurovascular contact area, the volume of radiation that single isocenter GKRS can provide may not be enough. Therefore, in order to better balance the effect of pain-control and the complications, our center routinely performed the two isocenters GKRS treatment for VBD-TN. Although the incidence of postoperative facial numbness is higher, only a small part of them are very annoying numbness[49].

Due to differences in technology, research methods, and patient selection among different institutions, the reported curative effect of surgical treatment for VBD-TN varies greatly. We, in this study, introduced the long-term experience of the surgical outcome based on a single institution using MVD and GKS for VBD-TN and compared their curative effect. To the knowledge of studies, this study was also the first report comparing two different treatments of VBD-TN. We found that initial and long-term favorable outcomes were higher in patients treated with MVD. In addition, we also found that patients treated with GKRS have a higher age compared with patients treated with MVD, which means that they are more likely to have underlying diseases and physical weakness. Although age indicator is not specifically used to determine whether to perform the MVD method, they do reflect that patients and doctors jointly carry out potential preference selection in order to obtain excellent pain-control results and a reasonable incidence of adverse events.

The incidence of favorable outcomes in the MVD group in this study was great (97.8\%), the rate of good pain-control at 7 years after surgery was $74.2 \%$, which was similar to previously reported results.

Furthermore, the transposition technique, consistent with our previous report[9], is a positive predictor of postoperative pain control. In the GKRS group, the incidence of early favorable outcomes was $78.9 \%$, and the rate of good pain-control at 7 years after surgery was $47.2 \%$, which is similar to the reported results by Chang et al[38] but better than some other studies. All 4 cases treated with GKRS, reported by Lorenzoni et al[26], had failed outcomes. Park et al[37] reported that the 1,2, and 5-year pain-relief rates for GKRS in VBD-TN patients remained at $53 \%, 38 \%$, and $10 \%$. The difference in treatment outcomes may be due to the two isocenters and multiple isocenters irradiation used in our and Chang's studies, respectively. Increasing the number of target points can cover a larger area of the affected myelinated nerve roots to increase the success rate of treatment, which is similar to the GKRS treatment for TN secondary to brain tumors. Most studies have shown that postoperative facial numbness is a positive predictor of pain relief[18, 20], which was confirmed by our studies. The occurrence of postoperative facial dysfunction in the MVD cohort does not seem to be related to the outcome. In addition, age and duration of symptoms were not statistically different between patients with good pain-control and patients with poor pain- 
control in the two groups, although some studies have shown that older age[32, 41] and shorter duration of symptoms was a predictor of postoperative pain relief[19, 48].

In terms of the time to obtain favorable outcomes, the MVD group was significantly more rapid and better than the GKRS group $(P=0.005)$, for which the completely different principles of the two technologies for relieving neurovascular conflicts. MVD surgery maintains a space between the trigeminal nerve and the responsible blood vessel, which can effectively interrupt the transmission of pulse force and free the trigeminal nerve from any contact. Thus eliminating the abnormal discharge caused by the formation of a short circuit between the blood vessel and the exposed myelin axon and stopping the onset of pain[31, 46]. The principle of two isocenters GKRS is not only to conform to the trigeminal nerve root but also to increase the irradiated volume of the trigeminal nerve, so that the axons are fully degenerated and decomposed. This process takes a certain amount of time to achieve, so it does not have the immediate effect of MVD. In addition, failure of positioning, insufficient radiation, and tight neurovascular conflicts will also affect the pain relief effect.

When it comes to complications, the MVD group has a variety of types compared to the GKRS group, but the overall complication incidence is significantly lower. The results were understood very well by us. MVD surgery is invasive, especially the intricate neurovascular structure in the cerebellopontine area, which can easily cause side damage to nerves and blood vessels during the operation. New or worsening facial numbness and hearing loss are the most common complications in the MVD group. The former was likely to be caused by the continuous traction of the trigeminal nerve during the operation, and the latter may be the retraction of the cerebellum that stretched the auditory nerve or caused by the new mechanical compression of the auditory nerve. These complications were not found in the GKRS group, and the most common complication was facial numbness, which is related to the working principle of the gamma knife. GKRS, destroyed the structural integrity of the trigeminal nerve through diffuse demyelination, increases the pain threshold and slows down the conduction of electrographic signals to relieve pain, but sometimes it is inevitable to damage non-pain sensory fibers[12]. The occurrence of postoperative complications may affect the quality of life of the patient. Therefore, for safe and effective treatment of VBD-TN, a skilled surgeon, required to perform rigorous preoperative evaluation, accurate target positioning, and appropriate radiation dose, is the key element to ensure the treatment effects and reduce the number of postoperative complications.

\section{Limitations}

This study certainly had some limitations. First, the present study was utilized a single-center retrospective design, thus recall bias might arise owing to the study design. Second, the patients were divided into two groups nonrandomly; there was a selection bias for the surgical method used. Patients who are older or in poor physical condition tend to be treated with GKRS. Third, there are differences in age between the MVD and GKRS cohorts in our study, although we found no statistical difference in age between patients with favorable outcomes and unfavorable outcomes in two cohorts, we still cannot rule out the possibility that the age difference may lead to the observed difference between the MVD group 
and the GKGS group. Fourth, the small sample size was not universally representative for all patients with VBD-TN.

\section{Conclusion}

The results of this study showed that MVD could provide superior treatment effects than two isocenters GKRS for patients with VBD-TN in terms of initial favorable outcomes and long-term pain control. Safety speaking, the GKRS with fewer serious postoperative complications is superior to MVD despite the higher incidence of facial numbness after GKRS. Certainly, this is a retrospective study with inevitable limitations, a randomized controlled trial with a large sample size would be required to compare MVD and two isocenters GKRS in the future.

\section{Declarations}

Funding: Not applicable.

Conflicts of interest/Competing interests: The authors declare no conflict of interest.

Availability of data and material: The data used to support the findings of this study are available from the corresponding author upon request.

Code availability: Not applicable

Ethics statement: The study received full approval from the ethics committee of Union Hospital, Tongji Medical College, Huazhong University of Science and Technology and informed consent was waived due to the retrospective nature of the study.

Consent to participate: Written informed consent for participate was obtained from all participants.

Consent for publication: Written informed consent for publication was obtained from all participants.

Authors' contributions: Study concept and design: Zhen Zhao and Songshan Chai. Data acquisition and analysis: Zhen Zhao and Jia-jing Wang. Writing the first draft: Zhen Zhao and Songshan Chai. Revision: Xiaobing Jiang and Chuansheng Nie. Supervision: Wei Xiang. Read and approved final version: all authors.

Acknowledgements: We would like to express my gratitude to the Linlin Gou for providing the hand-drawn operation pictures.

\section{References}

1. Alpert TE, Chung CT, Mitchell LT, Hodge CJ, Montgomery CT, Bogart JA, Kim DY, Bassano DA, Hahn SS (2005) Gamma knife surgery for trigeminal neuralgia: improved initial response with two 
isocenters and increasing dose. J NEUROSURG 102 Suppl:185-188.

https://doi.org/10.3171/jns.2005.102.s_supplement.0185

2. Al-Quliti KW (2015) Update on neuropathic pain treatment for trigeminal neuralgia. The pharmacological and surgical options. Neurosciences (Riyadh) 20:107-114.

https://doi.org/10.17712/nsj.2015.2.20140501

3. Alvarez-Pinzon AM, Wolf AL, Swedberg HN, Barkley KA, Cucalon J, Curia L, Valerio JE (2017) Comparison of Percutaneous Retrogasserian Balloon Compression and Gamma Knife Radiosurgery for the Treatment of Trigeminal Neuralgia in Multiple Sclerosis. WORLD NEUROSURG 97:590-594. https://doi.org/10.1016/j.wneu.2016.10.028

4. Apra C, Lefaucheur JP, Le Guerinel C (2017) Microvascular decompression is an effective therapy for trigeminal neuralgia due to dolichoectatic basilar artery compression: case reports and literature review. NEUROSURG REV 40:577-582. https://doi.org/10.1007/s10143-017-0812-5

5. Arrese I, Sarabia R (2016) Microvascular decompression for trigeminal neuralgia secondary to vertebrobasilar dolichoectasia. Case report, literature review, and pooled case analysis. Neurocirugia (Astur) 27:304-309. https://doi.org/10.1016/j.neucir.2016.05.003

6. Asplund P, Linderoth B, Bergenheim AT (2010) The predictive power of balloon shape and change of sensory functions on outcome of percutaneous balloon compression for trigeminal neuralgia. $J$ NEUROSURG 113:498-507. https://doi.org/10.3171/2010.2.JNS091466

7. Barker FN, Jannetta PJ, Bissonette DJ, Larkins MV, Jho HD (1996) The long-term outcome of microvascular decompression for trigeminal neuralgia. N Engl J Med 334:1077-1083. https://doi.org/10.1056/NEJM199604253341701

8. Bederson JB, Wilson CB (1989) Evaluation of microvascular decompression and partial sensory rhizotomy in 252 cases of trigeminal neuralgia. J NEUROSURG 71:359-367. https://doi.org/10.3171/jns.1989.71.3.0359

9. Chai S, Xu H, Wang Q, Li J, Wang J, Wang Y, Pool H, Lin M, Xiong N (2020) Microvascular decompression for trigeminal neuralgia caused by vertebrobasilar dolichoectasia: interposition technique versus transposition technique. Acta Neurochir (Wien) 162:2811-2821. https://doi.org/10.1007/s00701-020-04572-7

10. Chai S, Xu H, Xiao D, Wang J, Wang Y, Li J, Fu P, Pool H, Xiong N (2020) Salvage gamma knife surgery for recurrent glossopharyngeal neuralgia following microvascular decompression: a retrospective case series. Acta Neurochir (Wien). https://doi.org/10.1007/s00701-020-04654-6

11. Chang CS, Huang CW, Chou HH, Lin LY, Huang CF (2018) Outcome of Gamma Knife radiosurgery for trigeminal neuralgia associated with neurovascular compression. J CLIN NEUROSCI 47:174-177. https://doi.org/10.1016/j.jocn.2017.09.016

12. Chen JF, Lee ST (2003) Comparison of percutaneous trigeminal ganglion compression and microvascular decompression for the management of trigeminal neuralgia. Clin Neurol Neurosurg 105:203-208. https://doi.org/10.1016/s0303-8467(03)00012-x 
13. Dhople AA, Adams JR, Maggio WW, Naqvi SA, Regine WF, Kwok Y (2009) Long-term outcomes of Gamma Knife radiosurgery for classic trigeminal neuralgia: implications of treatment and critical review of the literature. Clinical article. J NEUROSURG 111:351-358.

https://doi.org/10.3171/2009.2.JNS08977

14. Elaimy AL, Lamm AF, Demakas JJ, Mackay AR, Lamoreaux WT, Fairbanks RK, Pfeffer RD, Cooke BS, Peressini BJ, Lee CM (2013) Gamma knife radiosurgery for typical trigeminal neuralgia: An institutional review of 108 patients. Surg Neurol Int 4:92. https://doi.org/10.4103/2152-7806.115163

15. El-Ghandour NM (2010) Microvascular decompression in the treatment of trigeminal neuralgia caused by vertebrobasilar ectasia. NEUROSURGERY 67:330-337. https://doi.org/10.1227/01.NEU.0000371978.86528.60

16. Hakanson S (1981) Trigeminal neuralgia treated by the injection of glycerol into the trigeminal cistern. NEUROSURGERY 9:638-646. https://doi.org/10.1227/00006123-198112000-00005

17. Harsh GT, Wilson CB, Hieshima GB, Dillon WP (1991) Magnetic resonance imaging of vertebrobasilar ectasia in tic convulsif. Case report. J NEUROSURG 74:999-1003.

https://doi.org/10.3171/jns.1991.74.6.0999

18. Kano H, Kondziolka D, Yang HC, Zorro O, Lobato-Polo J, Flannery TJ, Flickinger JC, Lunsford LD (2010) Outcome predictors after gamma knife radiosurgery for recurrent trigeminal neuralgia. NEUROSURGERY 67:1637-1645. https://doi.org/10.1227/NEU.0b013e3181fa098a

19. Karam SD, Tai A, Wooster M, Rashid A, Chen R, Baig N, Jay A, Harter KW, Randolph-Jackson P, Omogbehin A, Aulisi EF, Jacobson J (2014) Trigeminal neuralgia treatment outcomes following Gamma Knife radiosurgery with a minimum 3-year follow-up. J Radiat Oncol 3:125-130. https://doi.org/10.1007/s13566-013-0134-3

20. Kondziolka D, Zorro O, Lobato-Polo J, Kano H, Flannery TJ, Flickinger JC, Lunsford LD (2010) Gamma Knife stereotactic radiosurgery for idiopathic trigeminal neuralgia. J NEUROSURG 112:758765. https://doi.org/10.3171/2009.7.JNS09694

21. Kraemer JL, Pereira FAA, David G, Faria MB (2006) Vertebrobasilar dolichoectasia as a cause of trigeminal neuralgia: the role of microvascular decompression. Case report. Arq Neuropsiquiatr 64:128-131. https://doi.org/10.1590/s0004-282x2006000100027

22. Kurokawa Y, Maeda Y, Toyooka T, Inaba K (2004) Microvascular decompression for hemifacial spasm caused by the vertebral artery: a simple and effective transposition method using surgical glue. Surg Neurol 61:398-403. https://doi.org/10.1016/S0090-3019(03)00425-7

23. Lin CF, Chen HH, Hernesniemi J, Lee CC, Liao CH, Chen SC, Chen MH, Shih YH, Hsu SP (2012) An easy adjustable method of ectatic vertebrobasilar artery transposition for microvascular decompression. Clin Neurol Neurosurg 114:951-956. https://doi.org/10.1016/j.clineuro.2012.02.021

24. Linskey ME, Jho HD, Jannetta PJ (1994) Microvascular decompression for trigeminal neuralgia caused by vertebrobasilar compression. J NEUROSURG 81:1-9. https://doi.org/10.3171/jns.1994.81.1.0001 
25. Liu J, Chen Z, Feng T, Jiang B, Yuan Y, Yu Y (2019) Biomedical Glue Sling Technique in Microvascular Decompression for Trigeminal Neuralgia Caused by Atherosclerotic Vertebrobasilar Artery: A Description of Operative Technique and Clinical Outcomes. WORLD NEUROSURG 128:e74e80. https://doi.org/10.1016/j.wneu.2019.03.289

26. Lorenzoni JG, Massager N, David P, Devriendt D, Desmedt F, Brotchi J, Levivier M (2008) Neurovascular compression anatomy and pain outcome in patients with classic trigeminal neuralgia treated by radiosurgery. NEUROSURGERY 62:368-375. https://doi.org/10.1227/01.neu.0000316003.80893.81 375-376.

27. Love S, Coakham HB (2001) Trigeminal neuralgia: pathology and pathogenesis. BRAIN 124:23472360. https://doi.org/10.1093/brain/124.12.2347

28. Lye RH (1986) Basilar artery ectasia: an unusual cause of trigeminal neuralgia. J Neurol Neurosurg Psychiatry 49:22-28. https://doi.org/10.1136/jnnp.49.1.22

29. Ma X, Sun X, Yao J, Ni S, Gong J, Wang J, Li X (2013) Clinical analysis of trigeminal neuralgia caused by vertebrobasilar dolichoectasia. NEUROSURG REV 36:573-578. https://doi.org/10.1007/s10143-013-0468-8

30. Maarbjerg S, Gozalov A, Olesen J, Bendtsen L (2014) Trigeminal neuralgia-a prospective systematic study of clinical characteristics in 158 patients. HEADACHE 54:1574-1582. https://doi.org/10.1111/head.12441

31. Marchan EM, Vender J, Cantrell RR, Alleyne C, Norris C, Madden N, Marascio JA, Rabatic BM, Zaenger D, Al-Basheer A, Aletan M, Amoush A, Pishgou M, Ferguson CL, Huang K, Shaaban S, Green A, Mott F, Figueroa RE, Mourad WF (2016) The Role of Internationally Standardized Radiosurgery Treatment Parameters in Predicting Long-Term Trigeminal Neuralgia Outcomes. INT J RADIAT ONCOL 96S:S175-S176. https://doi.org/10.1016/j.jrobp.2016.06.442

32. Miller JP, Magill ST, Acar F, Burchiel KJ (2009) Predictors of long-term success after microvascular decompression for trigeminal neuralgia. J NEUROSURG 110:620-626. https://doi.org/10.3171/2008.9.17660

33. Miner ME, Rea GL, Handel S, Bertz J (1980) Trigeminal neuralgia due to dolichoectasia: angiographic and CT findings in a patient with the EEC syndrome. NEURORADIOLOGY 20:163-166. https://doi.org/10.1007/BF00341782

34. Miyazaki S, Fukushima T, Tamagawa T, Morita A (1987) [Trigeminal neuralgia due to compression of the trigeminal root by a basilar artery trunk. Report of 45 cases]. Neurol Med Chir (Tokyo) 27:742748. https://doi.org/10.2176/nmc. 27.742

35. Mousavi SH, Niranjan A, Huang MJ, Laghari FJ, Shin SS, Mindlin JL, Flickinger JC, Lunsford LD (2015) Early radiosurgery provides superior pain relief for trigeminal neuralgia patients. NEUROLOGY 85:2159-2165. https://doi.org/10.1212/WNL.0000000000002216

36. Nagaseki Y, Horikoshi T, Omata T, Ueno T, Uchida M, Nukui H, Tsuji R, Sasaki H (1992) Oblique sagittal magnetic resonance imaging visualizing vascular compression of the trigeminal or facial nerve. J NEUROSURG 77:379-386. https://doi.org/10.3171/jns.1992.77.3.0379 
37. Park KJ, Kondziolka D, Kano H, Berkowitz O, Ahmed SF, Liu X, Niranjan A, Flickinger JC, Lunsford LD (2012) Outcomes of Gamma Knife surgery for trigeminal neuralgia secondary to vertebrobasilar ectasia. J NEUROSURG 116:73-81. https://doi.org/10.3171/2011.8.JNS11920

38. Riesenburger RI, Hwang SW, Schirmer CM, Zerris V, Wu JK, Mahn K, Klimo PJ, Mignano J, Thompson CJ, Yao KC (2010) Outcomes following single-treatment Gamma Knife surgery for trigeminal neuralgia with a minimum 3-year follow-up. J NEUROSURG 112:766-771. https://doi.org/10.3171/2009.8.JNS081706

39. Somaza S, Hurtado W, Montilla E, Ghaleb J (2014) Gamma knife radiosurgery to the trigeminal ganglion for treatment of trigeminal neuralgia secondary to vertebrobasilar ectasia. Surg Neurol Int 5:S580-S585. https://doi.org/10.4103/2152-7806.148056

40. Sun S, Jiang W, Wang J, Gao P, Zhang X, Jiao L, Liu W, Shu K, Lei T (2017) Clinical analysis and surgical treatment of trigeminal neuralgia caused by vertebrobasilar dolichoectasia: A retrospective study. INT J SURG 41:183-189. https://doi.org/10.1016/j.ijsu.2017.04.015

41. Theodosopoulos PV, Marco E, Applebury C, Lamborn KR, Wilson CB (2002) Predictive model for pain recurrence after posterior fossa surgery for trigeminal neuralgia. Arch Neurol 59:1297-1302. https://doi.org/10.1001/archneur.59.8.1297

42. Tuleasca C, Carron R, Resseguier N, Donnet A, Roussel P, Gaudart J, Levivier M, Regis J (2014) Trigeminal neuralgia related to megadolichobasilar artery compression: a prospective series of twenty-nine patients treated with gamma knife surgery, with more than one year of follow-up. Stereotact Funct Neurosurg 92:170-177. https://doi.org/10.1159/000362172

43. Vanaclocha V, Herrera JM, Martinez-Gomez D, Rivera-Paz M, Calabuig-Bayo C, Vanaclocha L (2016) Is There a Safe and Effective Way to Treat Trigeminal Neuralgia Associated with Vertebrobasilar Dolichoectasia? Presentation of 8 Cases and Literature Review. WORLD NEUROSURG 96:516-529. https://doi.org/10.1016/j.wneu.2016.08.085

44. Waltz TA, Dalessio DJ, Ott KH, Copeland B, Abbott G (1985) Trigeminal cistern glycerol injections for facial pain. HEADACHE 25:354-357. https://doi.org/10.1111/j.1526-4610.1985.hed2507354.x

45. Wei Y, Pu C, Li N, Cai Y, Shang H, Zhao W (2018) Long-Term Therapeutic Effect of Microvascular Decompression for Trigeminal Neuralgia: Kaplan-Meier Analysis in a Consecutive Series of 425 Patients. TURK NEUROSURG 28:88-93. https://doi.org/10.5137/1019-5149.JTN.18322-16.1

46. Yadav S, Mittal HC, Sachdeva A, Verma A, Dhupar V, Dhupar A (2015) A retrospective study of 72 cases diagnosed with idiopathic trigeminal neuralgia in indian populace. J Clin Exp Dent 7:e40-e44. https://doi.org/10.4317/jced.51771

47. Zakrzewska JM, Linskey ME (2014) Trigeminal neuralgia. BMJ 348:g474. https://doi.org/10.1136/bmj.g474

48. Zeng YJ, Zhang H, Yu S, Zhang W, Sun XC (2018) Efficacy and Safety of Microvascular Decompression and Gamma Knife Surgery Treatments for Patients with Primary Trigeminal Neuralgia: A Prospective Study. WORLD NEUROSURG 116:e113-e117. https://doi.org/10.1016/j.wneu.2018.04.120 
49. Zhao H, Shen Y, Yao D, Xiong N, Abdelmaksoud A, Wang H (2018) Outcomes of Two-Isocenter Gamma Knife Radiosurgery for Patients with Typical Trigeminal Neuralgia: Pain Response and Quality of Life. WORLD NEUROSURG 109:e531-e538. https://doi.org/10.1016/j.wneu.2017.10.014

\section{Tables}

Table 1. Baseline characteristics of patients with VBD-TN in MVD group and GKS group

\begin{tabular}{|llll|}
\hline & MVD $(\mathrm{n}=46)$ & GKRS $(\mathrm{n}=34)$ & P value \\
\hline Mean age (years) & 61.1 & 65.4 & 0.03 \\
\hline Gender (F/\%) & $18(39.1 \%)$ & $10(29.4 \%)$ & 0.37 \\
\hline Pain laterality (L/\%) & $30(65.2 \%)$ & $20(58.8 \%)$ & 0.75 \\
\hline With prior operation, $\mathrm{n}(\%)$ & $7(15.2 \%)$ & $8(21.1 \%)$ & 0.35 \\
\hline Pain distribution & & & 0.47 \\
\hline Single branch (V1, V2 or V3) & $16(21.7 \%)$ & $9(26.5 \%)$ & \\
\hline V1+2 & $3(6.5 \%)$ & $2(5.9 \%)$ & \\
\hline V2+3 & $20(43.5 \%)$ & $13(38.2 \%)$ & \\
\hline V1+2+3 & $7(15.2 \%)$ & $10(29.4 \%)$ & 0.30 \\
\hline Duration of symptoms (years) & $3.8 \pm 2.3$ & $4.6 \pm 3.9$ & 0.17 \\
\hline Baseline BNI score, $n$ (\%) & & & 0.78 \\
\hline IV & $24(52.2 \%)$ & $12(35.3 \%)$ & \\
\hline V & $22(47.8 \%)$ & $22(64.7 \%)$ & \\
\hline Offending VBA (Radiological findings) & & & \\
\hline VA & $37(80.4 \%)$ & $26(76.5 \%)$ & \\
\hline BA & $9(19.6 \%)$ & $8(23.5 \%)$ & \\
\hline Arterial hypertension & $17(40.0 \%)$ & $17(50.0 \%)$ & 0.25 \\
\hline Median follow-up (months) & 61.1 & 56.8 & \\
\hline
\end{tabular}

F female; L left; VBA vertebrobasilar artery; VA vertebral artery; BA basilar artery; MVD microvascular decompression; GKRS Gamma Knife radiosurgery

Table 2. Pain outcomes and complications of patients with VBD-TN after MVD and GKRS 


\begin{tabular}{|c|c|c|c|}
\hline & MVD $(n=46)$ & GKRS (n = 34) & $\begin{array}{l}P \\
\text { value }\end{array}$ \\
\hline Initial pain relief, n (\%) & & & 0.005 \\
\hline 1 & $43(93.5 \%)$ & $24(70.1 \%)$ & \\
\hline II & $2(4.3 \%)$ & $3(8.8 \%)$ & \\
\hline III & $1(2.2 \%)$ & $3(2.9 \%)$ & \\
\hline IV & 0 & $1(40.0 \%)$ & \\
\hline V & 0 & $3(8.8 \%)$ & \\
\hline Initial favorable outcome (BNI I or II), n (\%) & $45(97.8)$ & $27(79.4)$ & 0.009 \\
\hline Median time to pain relief (IQR) & $\begin{array}{l}0 \text { day }(0-0 \\
\text { day) }\end{array}$ & $\begin{array}{l}8 w k s(1 w k- \\
6 \mathrm{mos})\end{array}$ & $<0.001$ \\
\hline New or worse numbness, $\mathrm{n}(\%)$ & $3(6.5 \%)$ & $16(47.1 \%)$ & $<0.001$ \\
\hline Not bothersome & $2(4.3 \%)$ & $12(35.3)$ & \\
\hline Somewhat bothersome & $1(2.2 \%)$ & $2(5.9)$ & \\
\hline Very bothersome & 0 & $2(5.9)$ & \\
\hline Other complications, n (\%) & & & 0.036 \\
\hline Newly development of dry eyes & 1 & 2 & - \\
\hline Facial palsy & 1 & 0 & - \\
\hline Taste hypoesthesia & 2 & 0 & - \\
\hline Hearing loss & 3 & 0 & - \\
\hline Diplopia & 1 & 0 & - \\
\hline CSF leakage & 1 & 0 & - \\
\hline Wound infection & 2 & 0 & - \\
\hline BNI score at last follow-up, n (\%) & & & 0.011 \\
\hline I & $36(78.3 \%)$ & $17(50.0 \%)$ & \\
\hline II & $2(4.3 \%)$ & $3(8.8 \%)$ & \\
\hline III & $2(4.3 \%)$ & $4(11.8 \%)$ & \\
\hline IV & $2(4.3 \%)$ & $4(11.8 \%)$ & \\
\hline V & $4(8.7 \%)$ & $6(17.6 \%)$ & \\
\hline $\begin{array}{l}\text { Favorable outcome (BNI I or II) at last follow-up, } n \\
(\%)\end{array}$ & $38(82.6 \%)$ & $20(58.8 \%)$ & 0.043 \\
\hline
\end{tabular}


MVD microvascular decompression; GKRS Gamma Knife radiosurgery

Table 3. Comparison of clinical factors between favorable outcomes and unfavorable outcomes in MVD group

\begin{tabular}{|c|c|c|c|}
\hline & $\begin{array}{l}\text { Favorable Outcome, BNI } \\
\text { I or II }\end{array}$ & $\begin{array}{l}\text { Unfavorable Outcome, } \\
\text { BNI III-V }\end{array}$ & $\begin{array}{l}P \\
\text { value }\end{array}$ \\
\hline No. of patients & 38 & 8 & \\
\hline Mean age (years) & 59.3 & 62.4 & 0.77 \\
\hline Gender (F/\%) & $14(36.8 \%)$ & $4(50.0 \%)$ & 0.69 \\
\hline Pain laterality, (L/\%) & $24(63.2 \%)$ & $6(75.0 \%)$ & 0.45 \\
\hline With prior operation, n (\%) & $5(13.2 \%)$ & $2(25.0 \%)$ & 0.59 \\
\hline Pain distribution & & & 0.93 \\
\hline Single branch (V1, V2 or V3) & $13(34.2 \%)$ & $2(25.0 \%)$ & \\
\hline $\begin{array}{l}\text { Multiple branch }(\mathrm{V} 1+2 ; \\
\text { V2+3; } 1+2+3)\end{array}$ & $25(65.8 \%)$ & $6(75.0 \%)$ & \\
\hline Duration of symptoms, (years) & $3.6 \pm 2.2$ & $4.0 \pm 2.5$ & 0.44 \\
\hline \multicolumn{4}{|l|}{ Baseline BNI score, n (\%) } \\
\hline IV & $21(55.3 \%)$ & $3(37.5 \%)$ & 0.45 \\
\hline V & $17(44.7 \%)$ & $5(62.5 \%)$ & \\
\hline $\begin{array}{l}\text { Offending VBA (Radiological } \\
\text { findings) }\end{array}$ & & & 0.95 \\
\hline VA & 30 (78.9\%) & $7(87.5 \%)$ & \\
\hline BA & $8(21.1 \%)$ & $1(12.5 \%)$ & \\
\hline Arterial hypertension & $13(34.2 \%)$ & $4(50.0 \%)$ & 0.66 \\
\hline MVD method & & & 0.037 \\
\hline Interposition technique & $15(39.5 \%)$ & $7(87.5 \%)$ & \\
\hline Transposition technique & $23(60.5 \%)$ & $1(12.5 \%)$ & \\
\hline $\begin{array}{l}\text { New or worse numbness post- } \\
\text { MVD, } \mathrm{n}(\%)\end{array}$ & $2(5.3 \%)$ & $1(12.5)$ & 1 \\
\hline
\end{tabular}

F female; L left; VBA vertebrobasilar artery; VA vertebral artery; BA basilar artery; MVD microvascular decompression 
Table 4. Comparison of clinical factors between favorable outcomes and unfavorable outcomes in GKRS group

\begin{tabular}{|c|c|c|c|}
\hline & $\begin{array}{l}\text { Favorable Outcome, BNI } \\
\text { I or II }\end{array}$ & $\begin{array}{l}\text { Unfavorable Outcome, } \\
\text { BNI III-V }\end{array}$ & $\begin{array}{l}\mathrm{P} \\
\text { value }\end{array}$ \\
\hline No. of patients & 20 & 14 & \\
\hline Mean age (years) & 66.1 & 64.2 & 0.77 \\
\hline Gender (F/\%) & $4(20.0 \%)$ & $6(42.9 \%)$ & 0.29 \\
\hline Pain laterality (L/\%) & $13(65.0 \%)$ & $7(50.0 \%)$ & 0.49 \\
\hline With prior operation, n (\%) & $4(25.0 \%)$ & $4(28.6 \%)$ & 0.69 \\
\hline Pain distribution & & & 0.70 \\
\hline Single branch (V1, V2 or V3) & $6(30.0 \%)$ & $3(21.4 \%)$ & \\
\hline $\begin{array}{l}\text { Multiple branch }(\mathrm{V} 1+2 \\
\text { V2+3;V1+2+3) }\end{array}$ & $14(70.0 \%)$ & $11(78.6 \%)$ & \\
\hline Duration of symptoms, (years) & $4.1 \pm 3.4$ & $4.8 \pm 3.9$ & 0.43 \\
\hline Baseline BNI score, n (\%) & & & 0.49 \\
\hline IV & $6(30.0 \%)$ & $6(42.9 \%)$ & \\
\hline V & $14(70.0 \%)$ & $8(57.1 \%)$ & \\
\hline $\begin{array}{l}\text { Offending VBA (Radiological } \\
\text { findings) }\end{array}$ & & & 1.00 \\
\hline VA & $15(75.0 \%)$ & $11(78.6 \%)$ & \\
\hline BA & $5(25.0 \%)$ & $3(21.4 \%)$ & \\
\hline Arterial hypertension & $8(40.0 \%)$ & $9(64.3 \%)$ & 0.73 \\
\hline GKRS dose & & & 0.72 \\
\hline 80 Gy & 0 & 1 & \\
\hline 84 Gy & 2 & 0 & \\
\hline 86 Gy & 3 & 1 & \\
\hline $88 \mathrm{~Gy}$ & 14 & 12 & \\
\hline $90 \mathrm{~Gy}$ & 1 & 0 & \\
\hline Median time to pain relief (IQR) & 7 wks (1 wk-6 mos) & 10 wks (0 day -7 mos) & 0.69 \\
\hline $\begin{array}{l}\text { New or worse numbness post- } \\
\text { GKRS, } n(\%)\end{array}$ & $13(65.0 \%)$ & $3(21.4 \%)$ & 0.031 \\
\hline
\end{tabular}


F female; L left; VBA vertebrobasilar artery; VA vertebral artery; BA basilar artery;

GKRS Gamma Knife radiosurgery

\section{Figures}

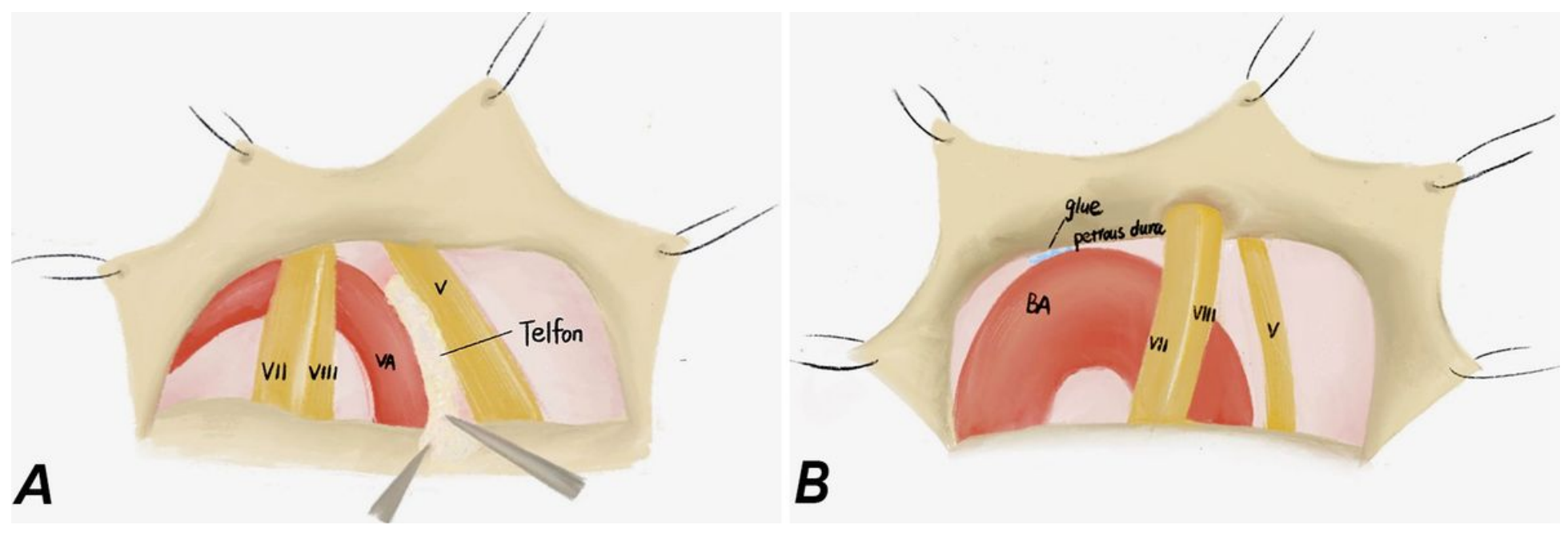

\section{Figure 1}

Intraoperative pictures of intervention method and transposition method. (A) Intervention method: the Teflon pad was inserted between the trigeminal nerve and VA; (B) Transposition method: the BA was fixed on the nearby petrous dura mater by using biological glue and a space was created between the trigeminal nerve and the BA.
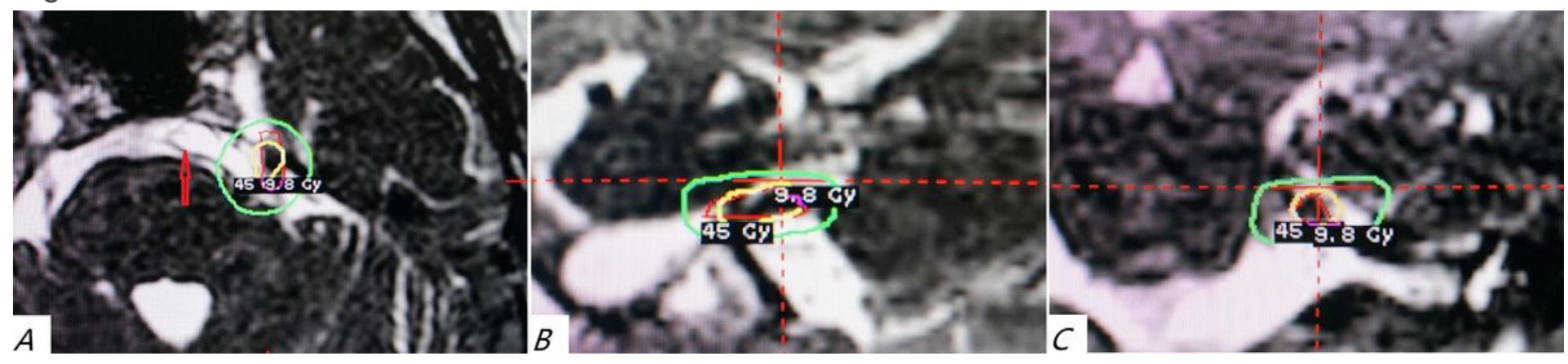

Figure 2

The two isocenters gamma knife irradiation on the trigeminal nerve of patients with VBD-TN are shown in the axial (A), sagittal (B) and coronal (C) positions, respectively. The red arrow shows the tortuous and dilated vertebrobasilar artery. The figure clearly shows the full length of the cisternal trigeminal nerve on the affected side, and shows the $50 \%$ isodose line ( $45 \mathrm{~Gy}$, yellow circle) and the isodose line of $9.8 \mathrm{~Gy}$ (green circle). 


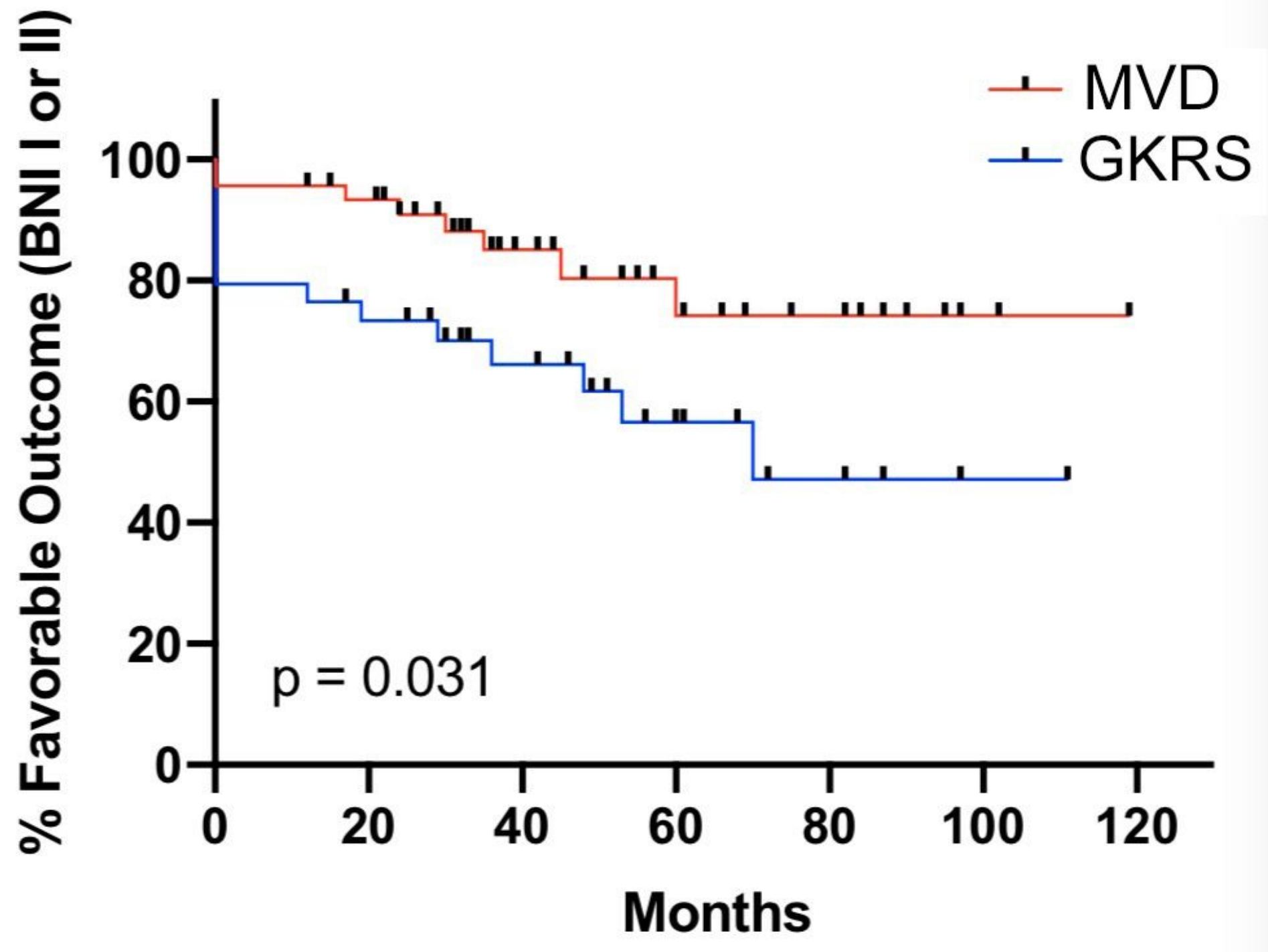

Figure 3

The Kaplan-Meier method calculated the ability of maintaining the postoperative favorable outcome (BNI $I-I)$ in the MVD group (red line) and GKRS (blue line) group ( $P=0.031)$. 